\title{
Una orientación terapéutica en la eclampsia
}

\section{Por LUIS GUILLERMO CUBLLLOS R.}

Bogotá, mayo 7 de 1953

Consigno $\mathrm{mi}$ reconocimiento muy sincero al señor Director de la Clínica Primero de Mayo, doctor José Vicente Sánchez Zaparta, quien en representación del I. C. S. S. aceptó e hizo posible la realización de esta nueva orienación terapéutica.

Señor Presidente y señores Miembros de la Sociedad Colombiana de Obstetricia y Ginecología.

Ciudad.

El 22 de abril de 1953 a la hora 22 y 40, ingresó al servicio de maternidad del I. C. S. S. Clínica Primero de Mayo. la paciente N. N. Registro No 19.599. carnet $N^{\circ}$ 01-088720, secundipara de 18 años en puerperio y eclampsia, habiéndome sido asignada por el Departamento de Admisión.

De los datos que me fueron sumlnistrados por el marido pude deducir:

1)-Que el primer embarazo tue de una evolución aparentemente normal, con parto y puerperto normales.

2)-Que el producto de la concepción de ese entonces, nació sano, en buenas condiciones y a término. Actualmente vive y siempre ha gozado de buena salud.
3)-Que tanto la evolución del segundo embarazo como del parto actual, fueron de apariencia normales. Sin embargo, el producto de la concepción ya en este caso nació prematuramente. Su peso al ingresarlo a la Clínica es de 2.080 gramos.

4)-Que este segundo parto se realizó en la casa a las 8 a. m. del día 22 de abril de 1953, es decir, el mismo díx de ingreso de la paciente a la clínica.

5)-Que al regresar el marido al hogar, después de su trabajo, halló a la enferma sin conocimiento, y presentando una serie de crisis convulsivas. motivo por el cual se vió obligado a solicitar la hospitalización de la paciente.

Al examen clínico se observa un estado de Coma Vigil, pues hay somnolencia, pérdida de la consciencia, y delirio. Pronuncia palabras incoherentes y se agita estando aparentemente dormida. De cuando en vez abre los ojos, al ser llamada. Pulso de 80 al minuto; temperatura de 38 grados; tensión arterial de $19 \times 11$. Se comprueba la realización de un parto completo $y$ reciente. No hay desgarros $n !$ incidenclas traumáticas 0 dinámicas consecuenciales al mismo. No se apreclan edemas, ni hay hemorragia genital. La pupila alterna de la midriasis 
a la miosis y aparecen crisis convulsivas tónicas y clónicas que se repiten sucesivamente en grupos de tres, cinco y luégo hasta diez, por lo cual se díagnostica: eclampsia del puerperio.

En estas condiciones, se ordena e: tratamiento médico modernamente preconizado por Bogen y Dieckmann. combinado las inyecciones de Sulfato de Magnesia, con grandes cantidades de solución glucosada al $33-1 / 3 \%$ vía endovenosa. Aplicamos dextrosa en vez de glucosa, por ser este el producto farmacéutico posible de adquirir en nuestro medio. Dicho tratamiento se condujo de la siguiente manera:

1)-Sulfato de Magnesia, solución inyectable al $20 \%, 40$ c. c. vía endovenosa.

2)-Dextrosa al 33-1/3\%, 40 c. c. vía endovenosa.

3)-Dextrosa al 10\%, 500 c. c. vía endovenosa, adicionándole Vitamina C., I gramo. Desgraciadamente no se pudieron aplicar en verdad sino 50 c. c. por dificultades con la enferma.

Este método terapéutico fue adicionado con la siguiente medicación:

\section{1) - Gardenal - Fenil-etil - malonil- úrea, 0.60 gramos, es decir, 3 am- polletas, vía intramuscular (sol. al $20 \%$ ).}

2)-Oxigenoterapia: a tensión de 4 litros.

Se procedió también a los cuidados accesorios de rutina, con la enferma como son: la atención por la lengua, la aspiración de mucosidades, el ais- lamiento, la fijación al lecho y el o curecimiento de la pieza, procurándo un medio tranquilo y de reposo. S extrajo con sonda una primera mue tra de orina, y se envió al laborator solicitando su examen parcial.

Dado que las crisis convulsive eclámpticas fueron aumentando pr gresivamento en númoro y on intons dad, haciéndose casi contmuas, an el tracaso de la medicación seguida la gravedad del pronóstico, llamé e junta médica al Profesor Santiago Ll ras Codarzi, quien después de exam nar cuidadosamente a la enferma, $c o r$ firmó el diagnóstico, juzgó muy gra el pronóstico recabando en el hect de tratarse de una eclampsia del pue perio y el carácter subintrante de crisis, aprobó la conducta seguide aconsejó continuar vigilando a la e ferma, y recurrir a las medidas ter péuticas que en cada momento consideraran como las más oportuno

Posteriormente tuve la convicci personal de que el cuadro clínico ca vez empeoraba, y que la medicaci parecía ineficaz. Por este motivo, té como norma terapéutica una co ducta nueva y personal mía, que cinco casos de clientela particular había demostrato su eficacia, a ber:

1)-Punción raquídea, dejando líquido céf́lalo-raquideo - de 15 20 c. c. - hasta una velocidad cana a la normal, seguida in diatamente de:

2)-Cafeína, una ampolleta de gramos, por vía intra-raquídeo

3)-Piridoxina clorhidrato, 0.50 gran vía endovenosa, $y, 0.75$ gras por vía intramuscular. 
4-Vitamina R. - Bisultito de Menadione y Sodio- 5 miligramos, y Normoclotín, 2 ampolletas, vía intramuscular.

Oxigenoterapia, como so venia nyli ando, a tension de 4 lts.

Exanguino-transfusión. S aplica: : wara este caso, 1.000 c. c. d. : wo total, y se extration 1.200 do sangre materna.

Las condiciones clínicas de la enterma mejoraron entonces rápida y progresivamente. Las crisis convulsivas tonicas y clónicas desaparecieron de manera rotunda cuando finalizaba ia exanguino-transfusión, y no volvieron a presentarse jamás. La tensión artera: descendió a $10 \times 7$, desde la hora día IV-23/53, manteniéndose estable, y la enferma, buscando de manera espontánea una posición más cómoda en el decúbito lateral izquierdo pidió que se la dejara dormir.

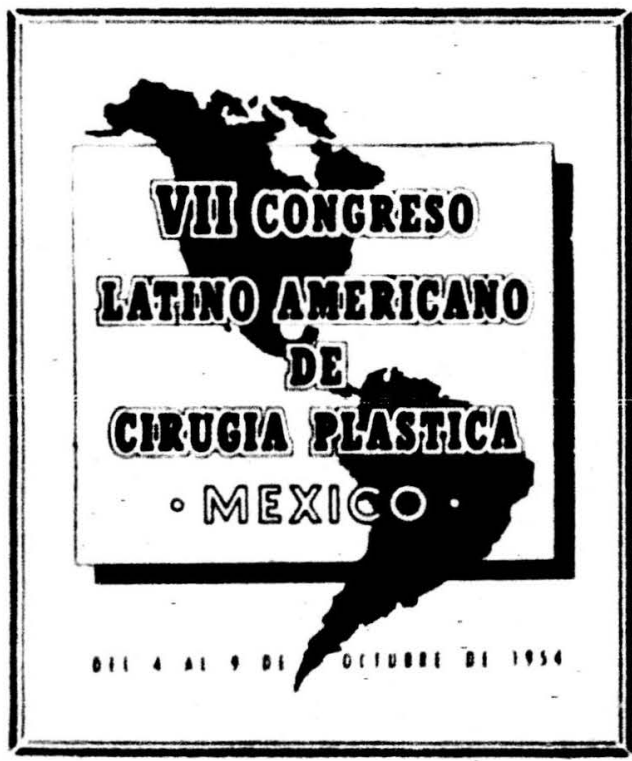

La evolución posterior tue en extremo satisfactoria, y ella queda plenamente consignada $y$ dimostrada en los cuadros adiuntos de sinopsis, que comprenden:

1) Sinopsis de la medicación posterior.

2) Sinopsis de laboratorio: Análisis de orina $y$ hematologia.

3)-Evolución de la tensión arterial.

Además, en el archivo oficial del I. C. S. S. -Clínica Primero de Mayo- reposa la historia clínica de la paciente bajo el registro número 19.599 , motivo de esta comunicación.

\section{Comentario}

La punción lumbar, o mejor, el drenaje lumbar, tiene una utilidad ya suficlentemente demostrada como medida terapéutica en la hipertensión del líquido céfalo-raquídeo, así como en el edema cerebral aqudo, cuyas sintomatologías parecen coincidir en ambos estados. Su práctica en la eclampsía es difícil, y requlere alguna experiencia. Tiene en cambio la ventaja de poder ser repetida y regulada de acuerdo con las circunstancias. Debo practicarse con método, y es necesario cuidar de no ir a provocar la estranqulación del bulbo sobre el reborde del aquiero occipital explicable por la presión de un encéfalo edematoso. caso de hallarse interrumpida la comunicación entre los espacios subaramoideos cerebrales y raquídeos.

La Cafoina Intra-raquidea, ha tentdo ya una vasta utilización para combatir diversos estados patológicos, y entre ellos, tal vez sea el más conoct- 
do el sindrome cefalálgico subsiguiente a la raquianestesia. Su mecanismo, aún no está suficientemente dilucidado, pero acaso él se deba a una acción miotónica circulatoria, coadyuvada por su acción paralizante sobre el gran simpático, antagonista de la adrenalina, y excitante por excelencia de! simpático como lo han demostrado las experiencias de Frederiq.

La piridoxina clorhidrats. o factor B6 del completo vitamínico $B$, ha demostrado su eficacia en algunas manifestaciones tóxicas del embarazo, tal vez por una acción sobre el metabolismo celular. Sin embargo, debemos reconocer que es necesario una investigación más a fondo en la intimidad de sus mecanismos químicos.

La exanguino-transfusión, hecha $\mathrm{co}$. . los rigores de la técnica. puede aceptarse como inocua, y goza a la vez de ser un poderoso desintoxicante para la paciente. Permite un juego fácil, útil y acaso muchas veces necesario con la tensión arterial de ia enferma, así como el transporte de un gran número de elementos favorables para los organismos fetal y materno. Puede ser repetida y regulada ai influjo de las circunstancias, y es necesario recalcar aqui, que su aplicación no es sólo útil en el tratamiento curativo de la eclampsia, sino también como medida $y$ norma preventiva en el tratamiento a fondo de las toxicosis gravídicas, y en especial, de la pre-sclampsia.

Ahora bien, si hemos de considerar que los organismos maternos sometidos a la iniluencia de la gestación laboran productos biológicos, como an- ti-cuerpos, etc., para mantener su equilibrio, cabe prejuzgar un mayor beneficio recolectando para estos fines, sangres de mujeres gestantes o en lactancia.

No por sencillo pudiéramos olvidar aquí-recomendar como norma útil para el control de las tensiones arteriales, la sistematización de las horas, como dato básico, tomando con unos mismos aparatos la tensión en uno y otro brazo simultáneamente para buscar el valor promedio útil, correspondiente. Las tensiones accesorias, tomadas esporádicamente, tienen un carácter investigativo, más bien descubridor, que de verdadero control estadístico.

Sea este el momento también de expresar mis agradecimientos al personal subalterno de la Clínica Primero de Mayo, por su eficiente colaboración en el cuidado de esta paciente. y de manera especial, a los doctores Nicolás Maure y Alonso bautista.

\section{REFERENCIAS}

1) Acosta Hernando. Conferencias de Obstetricia.

2)-De Lee Greenhill. Principios y Práctica de Obstetricia.

3)-Pérez Manuel Luis. Tratado de Obstetricia.

4)-Eppinger. Patología de la permeablldad.

5)-Wotepp - Kuhnan- H. Schroeder. Las vitaminas.

6)-Botella Llusiá. Patología Obstétrica. 
COMENTARIOS SOBRE EL TRABAJO DEL DOCTOR CUBILLOS

- Doctor Fernández: ¿Qué otros exámenes de laboratorio se practicaron a la enterma?

- Doctor Cubillos: Los de rutina. E3 de especial mención la dosificación de chlhumina en la orina que dió en el primot examen 1.080 gramos: en el segundo, practicado pocas horas después, 1.44 gramos, y en el tercero al dia siguiente, dió ligeras huellas.

-Doctor Fernández: Además de estos exámenes es necesario en estos casos controlar la ingestión y eliminación de líquidos y practicar las pruebas de funcionamiento renal.

Debe dosificarse también sistemáticamente la glicemia, pues en estos casos de toxemias es muy frecuente la hipoglicemia. A la tasa de la glicemia se le da valor pronóstico y tiene importancia también entre la acción con la empírica administración que antes se hacía de insulina y que muchas veces provocaba shock insulínico hipogicénico.
- Doctor Tamayo: Es de suma importancia también, practicar la dosificación de la uricemia, que en estos casos se encuentra aumentada. Los norteamericanos le dan a esta tasa gran valor pronóstico $y$ basándose en ella, orientan el tratamiento.

- Doclor Fernández: También debe investigarse la saturación de oxígeno en la sangre, que en estos casos disminuye.

-Doctor Prada: Se obtuvo el feto vivo.

-Doctor Cubillos: Sí, nazió vivo. Además este fue un caso de eclampsia del puerperio.

-Doctor Silva Mojica: Felicito al Dr. Cubillos por su interesante trabajo; me parece lógico y racional el tratamiento usado por él y recomiendo a los socios hacer más observaciones al respecto, con el fin de elaborar un trabajo bien documentado que se pueda presentar en el próximo Congreso de Obstetricia y Ginecología.

\section{Señores Médicos:}

PREFIERAN, HASTA DONDE SEA POSIBLE, LOS PRODUCTOS DE LOS LABORATORIOS FARMACEUTICOS $Y$ DEMAS ENTIDADES QUE COLABORAN CON SUS ANUNCIOS EN LAS REVISTAS MEDICAS COLOM. BIANAS, ESTIMULANDO ASI NUESTRO PROGRESO CIENTIFICO. 


\section{Sinopsis de: Iraboratorio - Analisis de orina}

AÑ 1953

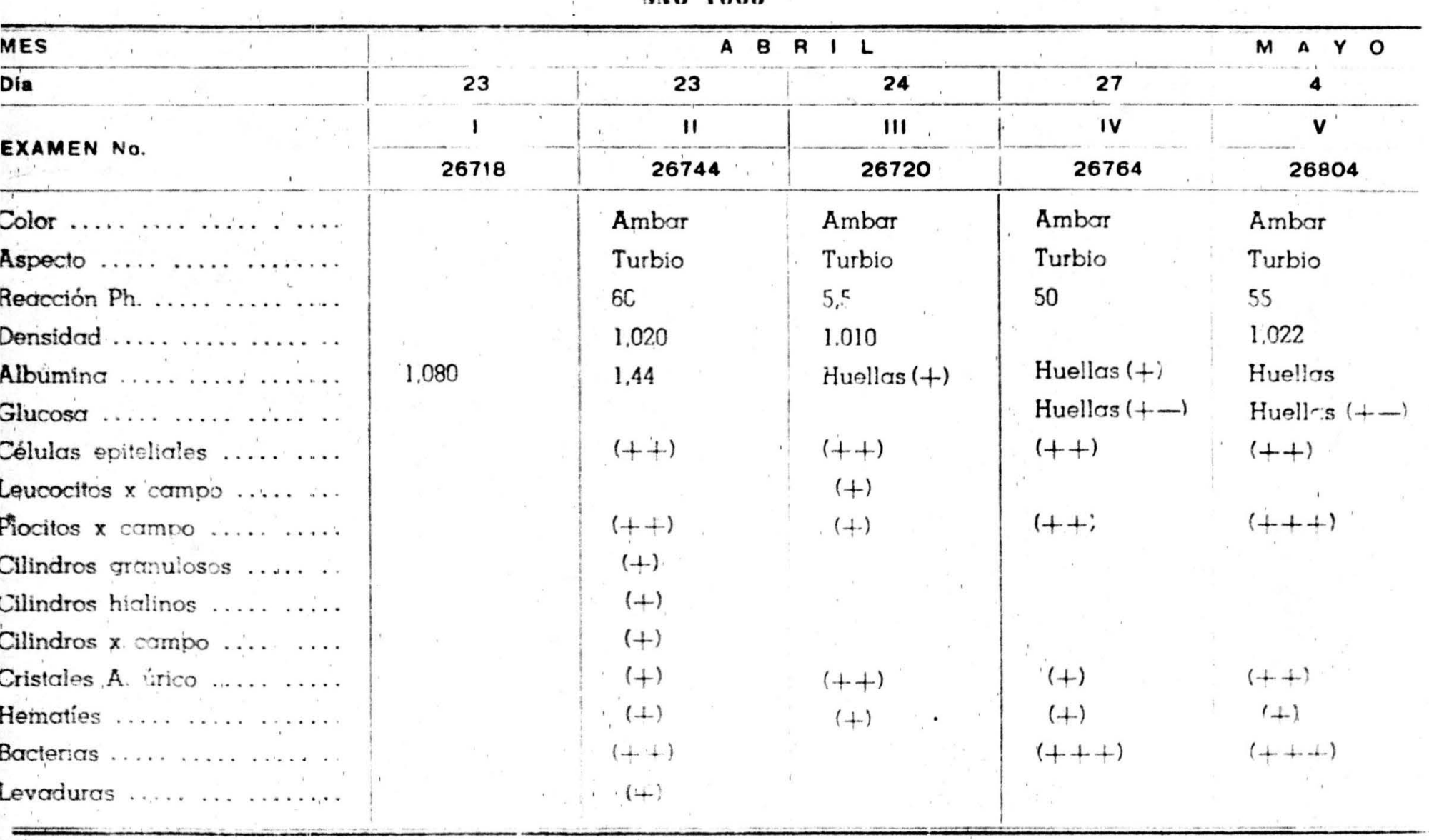




\section{Sinopsis de la Medicación Posterior}

\section{3}

\begin{tabular}{|c|c|c|c|c|c|c|c|c|c|c|c|c|c|}
\hline \multirow{2}{*}{, } & \multicolumn{8}{|c|}{ A B R $1 \mathrm{~L}$} & \multicolumn{5}{|c|}{ M A Y O } \\
\hline & 23 & 24 & 25 & 26 & 27 & 28 & 29 & 30 & 1 & 2 & 3 & 4 & 5 \\
\hline 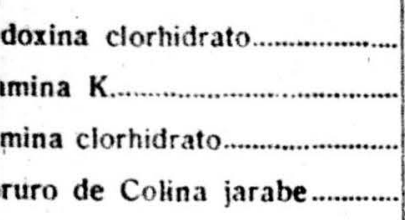 & $\begin{array}{l}0.50 \text { grs. } \\
2 \text { amps. }\end{array}$ & $\begin{array}{l}0.50 \mathrm{grs} . \\
2 \text { amps. }\end{array}$ & $\begin{array}{l}0.50 \mathrm{grs} \\
2 \text { amps. } \\
0.100 \mathrm{grs} \\
2 \text { cuch. }\end{array}$ & $\begin{array}{c}0.50 \mathrm{grs} \\
2 \text { amps. } \\
0.100 \mathrm{grs} \\
2 \text { cuch. }\end{array}$ & $\begin{array}{c}0.50 \mathrm{grs} \\
2 \text { amps. } \\
0.100 \mathrm{grs} . \\
2 \text { cuch. }\end{array}$ & 2 cuch. & 2 cuch. & $\begin{array}{l}0.100 \mathrm{grs} . \\
2 \text { cuch. }\end{array}$ & & & & & \\
\hline
\end{tabular}

\section{Sinopsis de otras Medidas Terapéuticas}

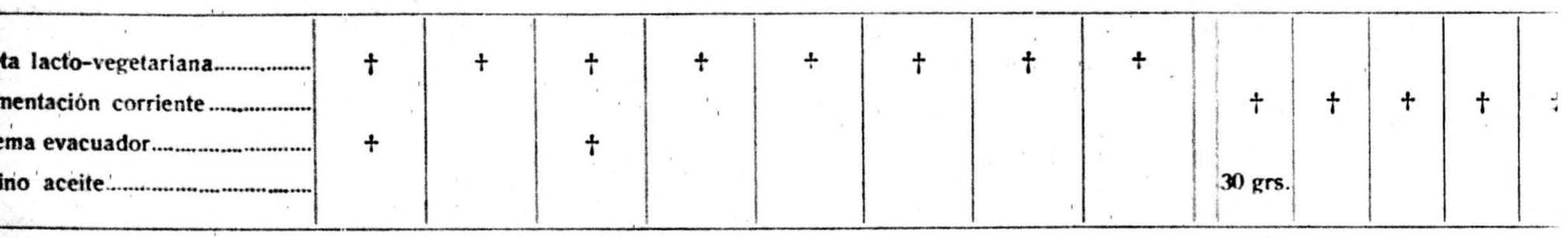




\section{Sinopsis de Taboratorio - Hermatología}

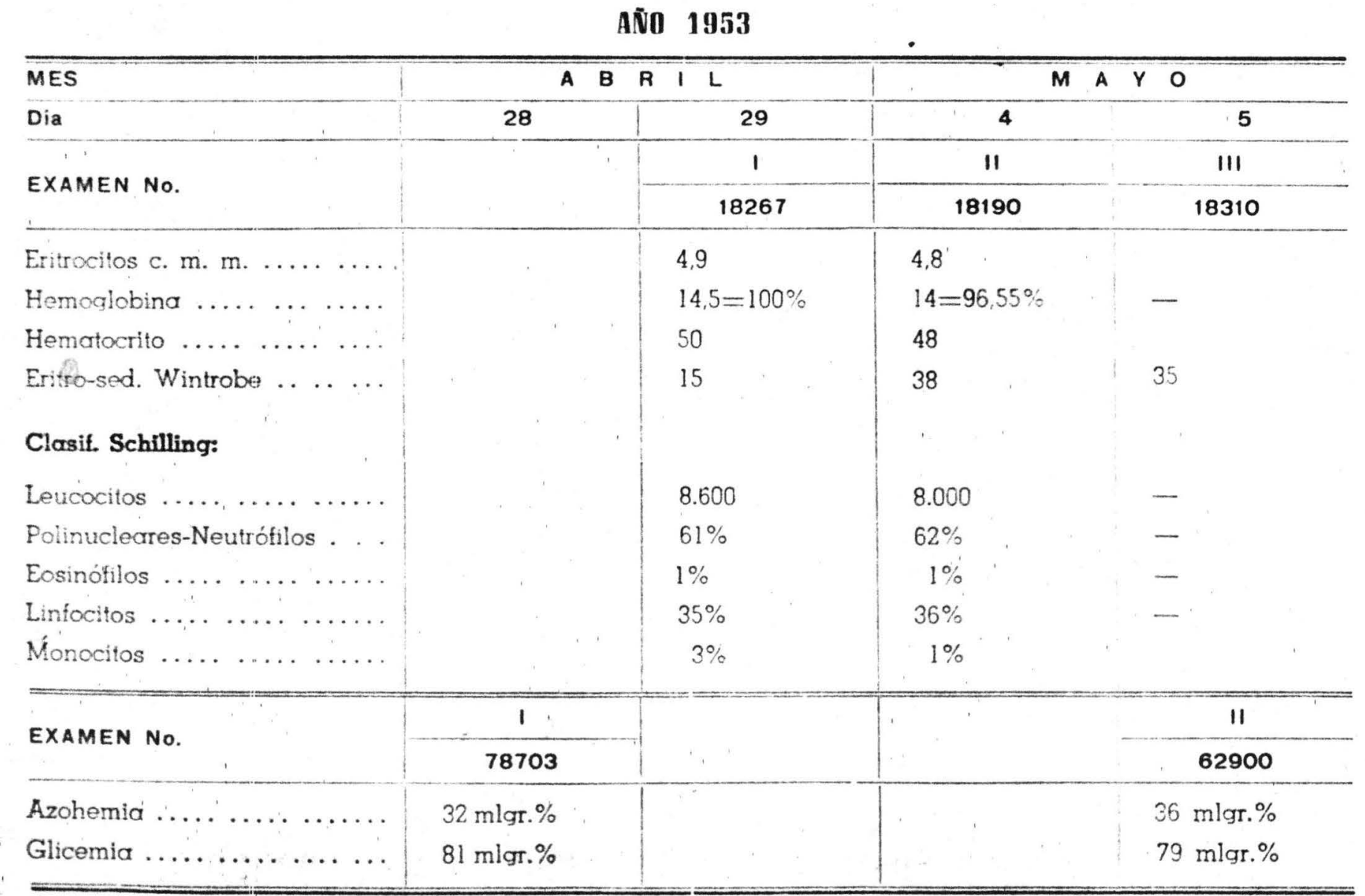




\section{fvolucion de la tensión arterial}

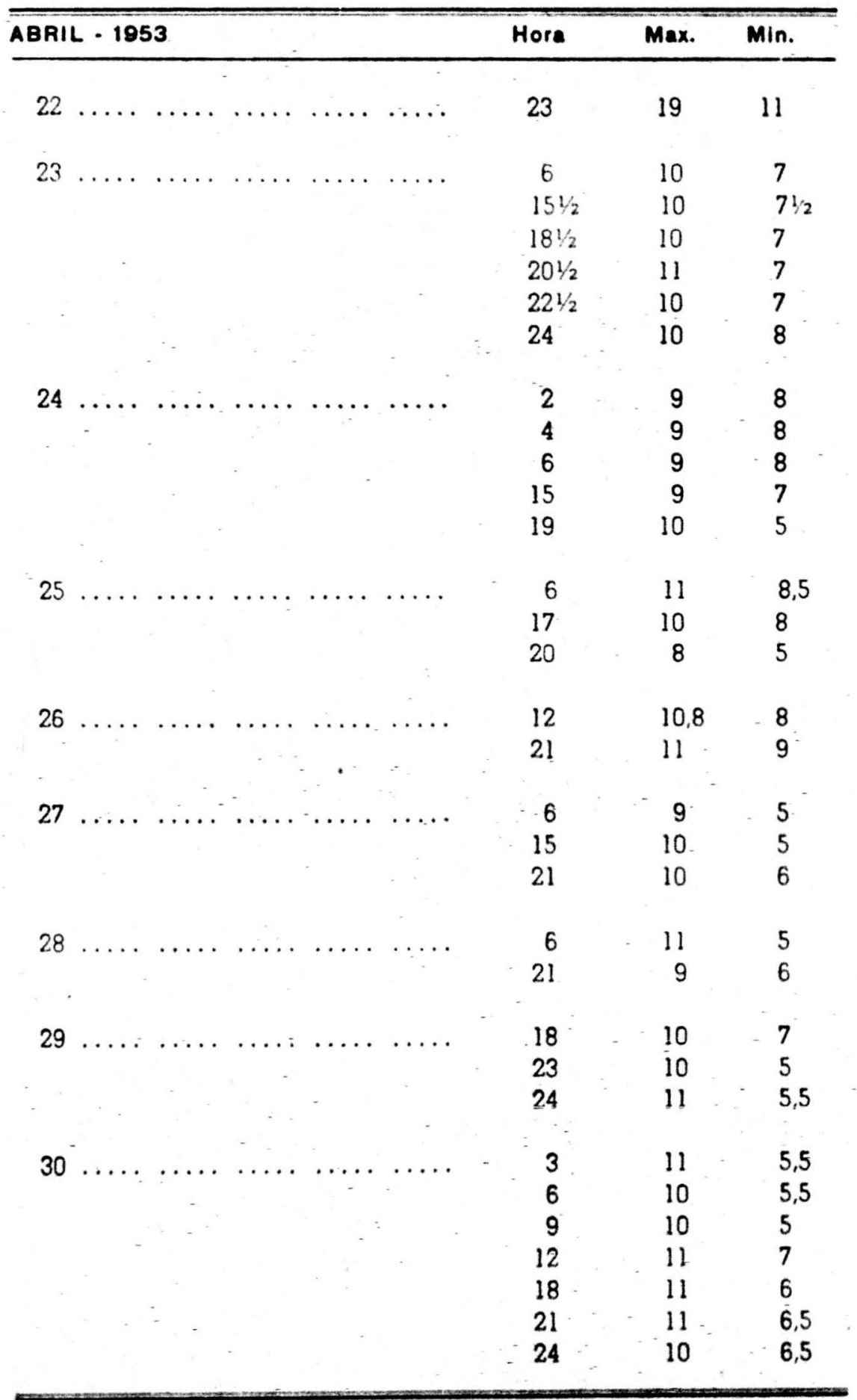

